\title{
Deep vein thrombosis due to May-Thurner syndrome: a case report
}

\author{
Yan Meng* (D), Hao Qin, Qiang Ma, Junbo Zhang, Bo Zhang, Honggang Pang, Qian Yin and Hongyan Tian
}

\begin{abstract}
Background: May-Thurner syndrome (MTS) or Cockett's syndrome is a rare clinical syndrome, which refers to the compression of the left common iliac vein (LCIV) by right common iliac artery and vertebral body. Complications of MTS include deep vein thrombus formation and even life-threatening pulmonary embolism.

Case presentation: Here, we report the case of a 60-year-old female patient with a complaint of swelling in the left lower limb and pain for 5 days. Computed tomography angiography indicated MTS, and thrombus formation of left external iliac vein and femoral vein. The patient was diagnosed with deep venous thrombosis (DVT) and MTS. The patient underwent ascending venography from the lower extremity to inferior vena cava (IVC) and then to the pulmonary artery with IVC filter implantation, left iliac vein balloon plasty, and stent placement. The patient visited the hospital for the removal of IVC filter, 28 days after the operation. After the interventional therapy, the patient had no in-stent restenosis and had remission during the 2-year follow-up.

Conclusions: This case presents a successful management of MTS in presence of DVT. Although clinicians are rarely aware, the presence of unilateral lower limb swelling and thrombosis may be the manifestations of MTS.
\end{abstract}

Keywords: May-Thurner syndrome, lliac vein plasty, Deep vein thrombosis, Endovascular management, Case report

\section{Background}

Deep venous thrombosis (DVT) is more common in the left side (60\%) than in the right side of the lower extremity [1]. Previous studies have revealed that this might be due to the compression of left common iliac vein (LCIV) by the right common iliac artery (RCIA). This condition is known as May-Thurner syndrome (MTS) [2]. This compression leads to the obstruction of venous outflow and increases the risk of DVT [3]. However, MTS presented variable clinical symptoms, which ranges from unilateral lower extremity swelling without thrombosis to minor cases of DVT [4].

Patients who have lower extremity DVT are at the risk of thrombus detachment and circulating it to the lung. This is known as pulmonary embolism (PE). For patients with contraindication to anticoagulant therapy,

\footnotetext{
* Correspondence: daxianghouzi@foxmail.com

Department of Peripheral Vascular Diseases, the First Affiliated Hospital of Xi'an Jiaotong University, No. 277 Yanta Road, Xi'an 710061, China
}

permanent or removable filters are placed in the inferior vena cava (IVC) to prevent PE [5]. For patients with both MTS and DVT, leg pain or swelling can be improved by stent placement [5].

In this report, a successful treatment experience of MTS in presence of DVT by the use of filter insertion, balloon plasty, and stent placement was discussed.

\section{Case presentation}

A 60-year-old female patient was hospitalized for swelling and pain in the left lower extremity for 5 days. It was reported that, 5 days ago, the patient experienced swelling of thigh, which gradually spread to the lower leg, and increased local skin tension and temperature. The patient had no fever, shortness of breath, chest pain, cough, expectoration, hemoptysis, amaurosis, or syncope; no special relevant medical, family, psycho-social past histories, and no history of chronic lower limb symptoms, even during her previous pregnancy. Deep 
vein ultrasound of the lower extremity at local hospital reported thrombosis of left common femoral vein, superficial femoral vein, deep femoral vein, popliteal vein, anterior tibial vein, posterior tibial vein, and intermuscular vein for the first time. She had no history of surgery or catheterization. On physical examination, her heart rate was $88 \mathrm{bpm}$, and blood pressure was 130/74 mmHg. Examination of the left lower limb revealed swelling and edema with moderate tenderness and pain, while the right lower limb was normal. Blood tests revealed normal blood routine and normal liver and kidney functions. Computed tomography angiography (CTA) indicated compression of LCIV by RCIA, narrowed LCIV lumen, thrombi formed from the femoral vein to left external iliac vein, and collateral circulation formed between bilateral external iliac vein (Fig. 1). She was diagnosed with DVT along with MTS.

The patient underwent IVC angiography, filter placement, left iliac vein plasty, and stent implantation under local anesthesia. The right femoral vein was punctured successfully with Seldinger technique [6] and then placed into the catheter sheath. Contrast agent was passed smoothly through IVC without filling defect (Fig. 2a). The sheath of the filter was transported, and a
Cordis Optease filter was placed in the IVC at second lumbar vertebrae (Fig. 2b), followed by puncturing of the left femoral vein. The catheter sheath was then inserted; angiography revealed severe stenosis of the left external iliac vein and the common iliac vein, and the pelvic collateral compensation was seen through contralateral reflux (Fig. 2c). A $14 \mathrm{~mm}-60 \mathrm{~mm}$ balloon was inserted into the IVC through a narrow segment of left external iliac vein and common iliac vein (Fig. 2d). Then, an E-lunimexx $14 \mathrm{~mm}-60 \mathrm{~mm}$ selfexpanding stent was inserted (Fig. 2e). The stent was in good position, and the expansion remained satisfactory (Fig. 2f). The contrast medium was passed through smoothly, and the original stenosis was relieved. At the end of the operation, the sheath of the bilateral inguinal area was removed, and the wound was dressed with a bandage. The patient returned to the ward safely and tolerated well, and was discharged 5 days after the surgery. The patient was prescribed with oral rivaroxaban and aspirin. After 28 days of the surgery, she came back to the hospital for the removal of IVC filter. This procedure was successful. The DVTs were ablated after 4 months of anticoagulation therapy.

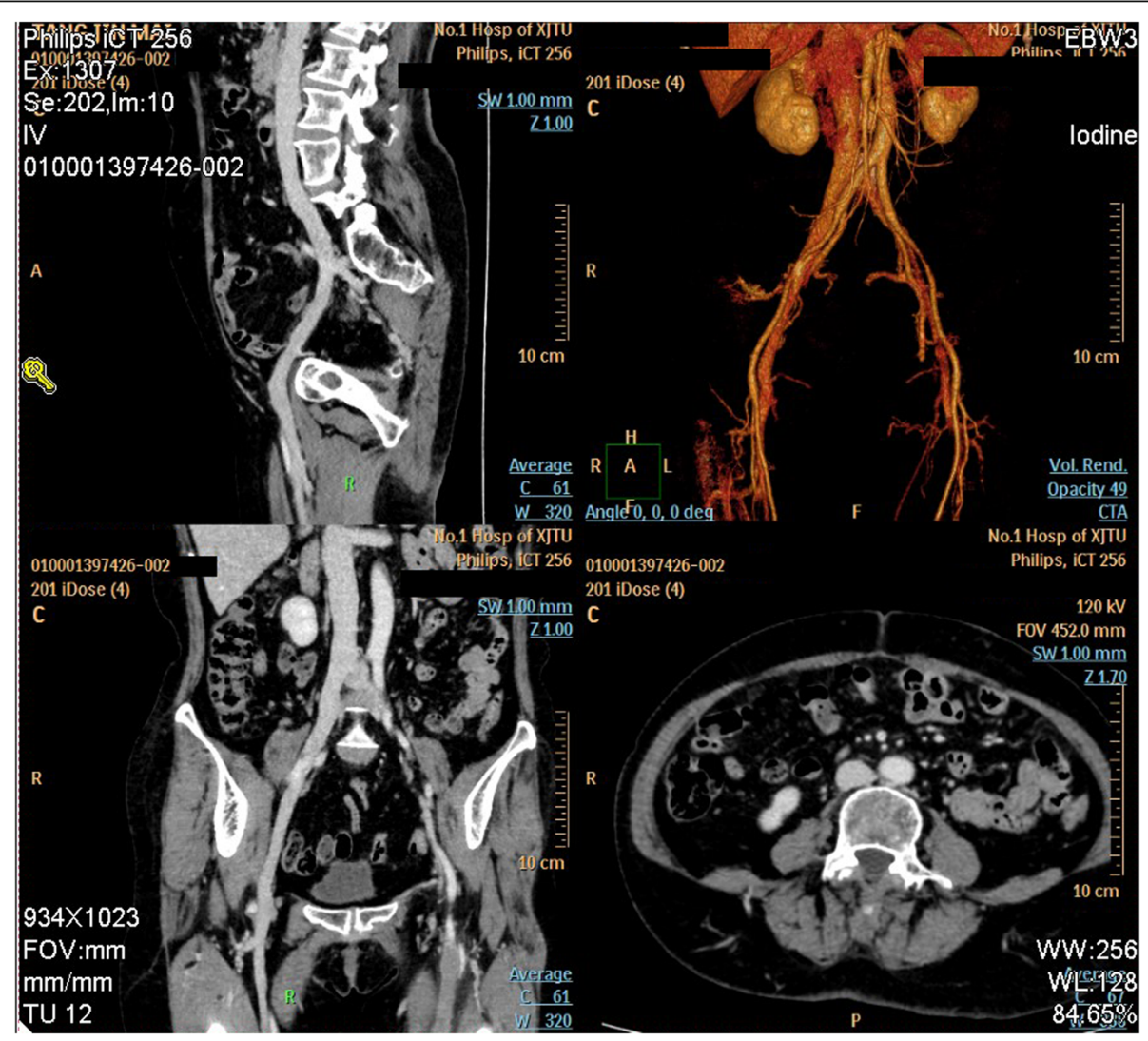

Fig. $1 C T$ arterial angiography before operation 


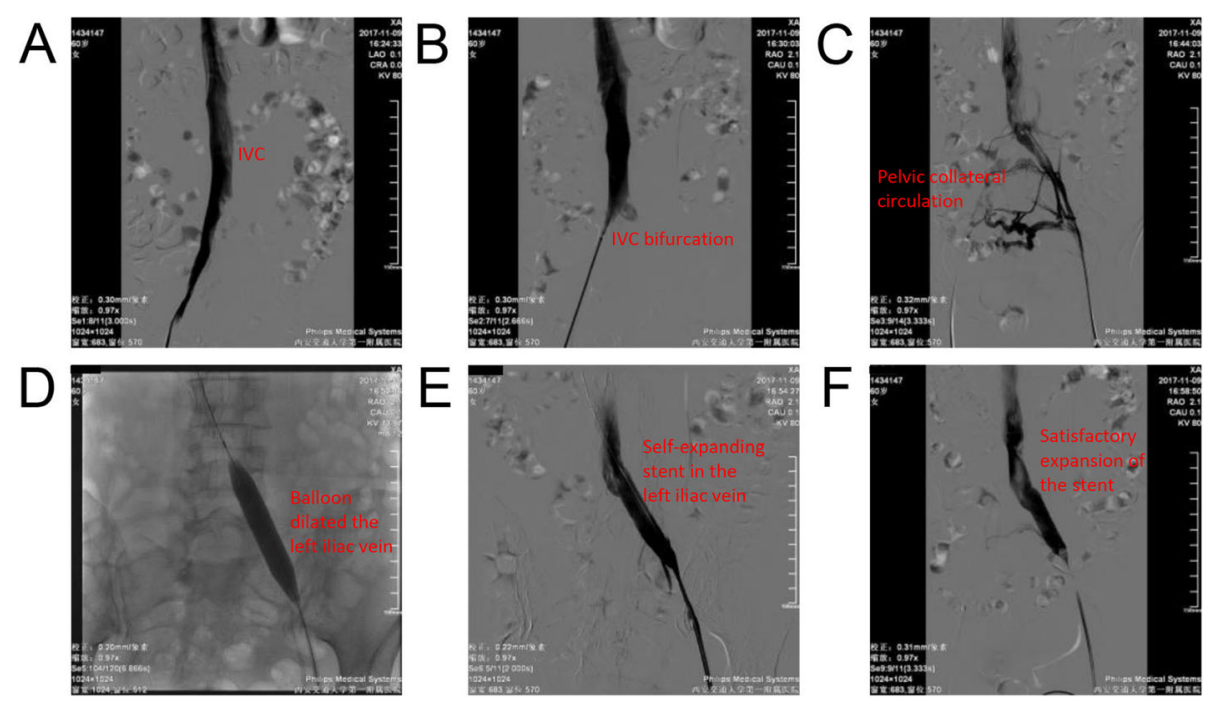

Fig. 2 Surgical procedure of venography. a Inferior vena cava angiography showed smooth blood flow. b Insertion of a Cordis Optease vena cava filter. $\mathbf{c}$ The left iliac vein was severely narrowed and the pelvic collateral circulation remained open. $\mathbf{d} 14 \mathrm{~mm}-60 \mathrm{~mm}$ balloon dilatation of left iliac vein. e An E-lunimexx $14 \mathrm{~mm}-60 \mathrm{~mm}$ self-expanding stent was implanted into the left iliac vein. $\mathbf{f}$ The stent was in good position, and the expansion remained satisfactory

\section{Discussion and conclusions}

May-Thurner syndrome was initially reported in 1956 by May and Thurner. They found that $22 \%$ of the studied adult subjects had thickened left iliofemoral venous wall, where the RCIA has crossed the LCIV. The obstruction was termed as a "spur," and this was equal to a callus formation under chronic repetitive stimulation from the compression of LCIV, by pulsating RCIA. Proliferation of cells at LCIV is the sequencing event, this might in turn aggravate stenosis and trigger venous thrombosis $[3,7]$. Previous studies have revealed that the incidence of LCIV compression ranged 14-32\% [8]; however, 2$5 \%$ of these patients complained of lower extremity symptoms [9]. MTS is more commonly observed in female patients than in male patients, and $18-49 \%$ of the MTS cases had left lower extremity DVT [10-12]. Moreover, pregnancy is a risk factor for DVT formation [13].

The diagnosis of MTS relies on imaging examinations. Certain tests should be conducted in patients with unilateral leg swelling to exclude MTS. Ultrasound could identify thrombus formation of DVT. However, in most of the patients, deep veins in the pelvis cannot be fully visualized by ultrasound [4]. If MTS is suspected with characteristic ultrasonography, further examination should be conducted by computed tomography venography (CTV) or magnetic resonance venography (MRV). Computed tomography venography and MRV are relatively noninvasive methods, which are used to visualize the deep veins in the pelvis. These methods can reveal the overlying of RCIA on LCIV, and the presence of compression and stenosis $[4,14]$. Another technique involves the combination of contrast venography and intravascular ultrasound, which is considered as a reliable approach for identifying abnormal venous blood flow and precisely locate the etiology and the degree of compression [4].

The traditional therapy for MTS is surgery and is outdated. The precise optimal treatment strategy remains to be determined. Endovascular treatments might be the first choice for effective management of MTS, which include techniques such as catheter-directed thrombolysis, IVC filter implantation, catheter-directed balloon angioplasty, and stenting placement $[5,13,15]$. Selective catheter-directed thrombolysis is considered effective for thrombus clearance and alleviation of symptoms [13]. IVC filter, which is also called as Greenfield filters, can efficiently prevent the spread of ruptured thrombus to the lungs [5]. Balloon angioplasty is often used in combination with stent placement and directly provides relief from anatomic stenosis of LCIV by the compression of RCIA [5]. According to a research in 39 patients with MTS, endovascular treatment showed $87 \%$ success rate [11].

In our study, the patient had acute swelling and pain in her left lower limb for 5 days. Computed tomography angiography showed DVT formation on the left external iliac vein and femoral veins, and obvious stenosis of LCIV due to the compression of RCIA. The patient underwent IVC filter placement, left iliac vein plasty, and stent implantation. The swelling of her left leg was largely alleviated after the operation, and she was in good condition without chronic symptoms of MTS for a two-year follow-up period. In MTS patients with only 6 months of warfarin antithrombotic therapy, the reported 
12-month stent patency rate was $78 \%$, while for the patients with more than 6 months of systemic anticoagulation or dual-antiplatelet therapy, the same was $89 \%$ [16]. At the time of discharge from the hospital, the patient had residual thrombi and was suggested to take rivaroxaban $10 \mathrm{mg}$ and aspirin $100 \mathrm{mg}$ once a day as long term anti-coagulation strategy, and to monitor coagulation function test, every 3 month in outpatient department.

Clinicians should be aware of the signs and symptoms of MTS and should consider this diagnosis in cases where the patients are presented with unexplained lower limb swelling or women with extensive left lower limb DVT during pregnancy.

Also, endovascular treatment appears to be a reliable therapy of MTS and can effectively alleviate the symptoms in patients.

\section{Abbreviations}

CTA: Computed tomography angiography; CTV: Computed tomography venography; MRV: Magnetic resonance venography; DVT: Deep vein thrombosis; MTS: May-Thurner syndrome; LCIV: Left common iliac vein; RCIA: Right common iliac artery; PE: Pulmonary embolism; IVC: Inferior vena cava

\section{Acknowledgements}

None.

\section{Authors' contributions}

YM and HQ designed the study; QM, JZ and BZ involved with patient care and contributed samples collection; YM, HP and QY analyzed the patient samples and interpreted data; YM wrote the initial paper; YM and HT revised the paper; YM had primary responsibility for final content. All authors read and approved the final manuscript.

\section{Funding}

This study was supported by the Fund for key research and development program project of Shaanxi province, China (No. 2017SF-254). The authors declare that the funding body was not involved in study design, data collection, analysis, interpretation and writing of the study.

\section{Availability of data and materials}

All data generated or analysed during this study are included in this published article.

\section{Ethics approval and consent to participate}

This study was approved by the Ethics Committee of the First Affiliated Hospital of Xi'an Jiaotong University. All procedures performed in studies involving human participants were in accordance with the ethical standards of the institutional and/or national research committee and with the 1964 Helsinki declaration and its later amendments or comparable ethical standards. Written informed consent was obtained from the individual participant included in the study.

\section{Consent for publication}

Written informed consent was obtained from the patient for publication of this case report and any accompanying images. A copy of the written consent is available for review by the Editor of this journal.

\section{Competing interests}

The authors declare that they have no competing interests.
Received: 3 March 2020 Accepted: 10 May 2020

Published online: 19 May 2020

\section{References}

1. Fazel R, Froehlich JB, Williams DM, Saint S, Nallamothu BK. Clinical problemsolving. A sinister development--a 35-year-old woman presented to the emergency department with a 2-day history of progressive swelling and pain in her left leg, without antecedent trauma. N Engl J Med. 2007:357:53-9

2. Narayan A, Eng J, Carmi L, McGrane S, Ahmed M, Sharrett AR, et al. lliac vein compression as risk factor for left- versus right-sided deep venous thrombosis: case-control study. Radiology. 2012;265:949-57.

3. May R, Thurner J. The cause of the predominantly sinistral occurrence of thrombosis of the pelvic veins. Angiology. 1957;8:419-27.

4. DeRubertis B, Patel R. May-Thurner syndrome: diagnosis and management. Curr Manage Venous Dis. 2018;1:463-77.

5. Carroll S, Moll S. Inferior vena cava filters, May-Thurner syndrome, and vein stents. Circulation. 2016;133:e383-7.

6. Higgs ZC, Macafee DA, Braithwaite BD, Maxwell-Armstrong CA. The Seldinger technique: 50 years on. Lancet. 2005;366:1407-9.

7. Cockett FB, Thomas ML, Negus D. lliac vein compression.--Its relation to iliofemoral thrombosis and the post-thrombotic syndrome. Br Med J. 1967;2:14-9

8. Ehrich WE, Krumbhaar EB. A frequent obstructive anomaly of the mouth of the left common iliac vein. Am Heart J. 1943;26:737-50.

9. Posner C, Owen M, Melhem N, Vidyarthi M, Low D, Renfrew I, et al. An acutely swollen leg. Clin Med (Lond). 2010;10:484-6.

10. Mickley V, Schwagierek R, Rilinger N, Gorich J, Sunder-Plassmann L. Left iliac venous thrombosis caused by venous spur: treatment with thrombectomy and stent implantation. J Vasc Surg. 1998;28:492-7.

11. O'Sullivan GJ, Semba CP, Bittner CA, Kee ST, Razavi MK, Sze DY, et al. Endovascular management of iliac vein compression (May-Thurner) syndrome. J Vasc Interv Radiol. 2000;11:823-36.

12. Oguzkurt L, Tercan F, Ozkan U, Gulcan O. lliac vein compression syndrome: outcome of endovascular treatment with long-term follow-up. Eur J Radiol. 2008;68:487-92.

13. Bozkaya H, Cinar C, Ertugay S, Korkmaz M, Guneyli S, Posacioglu H, et al. Endovascular treatment of iliac vein compression (May-Thurner) syndrome: angioplasty and stenting with or without manual aspiration Thrombectomy and catheter-directed thrombolysis. Ann Vasc Dis. 2015;8:21-8.

14. Mousa AY, AbuRahma AF. May-Thurner syndrome: update and review. Ann Vasc Surg. 2013;27:984-95

15. Budnur SC, Singh B, Mahadevappa NC, Reddy B, Nanjappa MC. Endovascular treatment of iliac vein compression syndrome (May-Thurner). Cardiovasc Interv Ther. 2013;28:101-5.

16. J. Padrnos L, Garcia D. May-Thurner syndrome and thrombosis: A systematic review of antithrombotic use after endovascular stent placement. Res Pract Thromb Haemost. 2018:3:70-8.

\section{Publisher's Note}

Springer Nature remains neutral with regard to jurisdictional claims in published maps and institutional affiliations.
Ready to submit your research? Choose BMC and benefit from:
- fast, convenient online submission
- thorough peer review by experienced researchers in your field
- rapid publication on acceptance
- support for research data, including large and complex data types
- gold Open Access which fosters wider collaboration and increased citations
- maximum visibility for your research: over $100 \mathrm{M}$ website views per year
At $\mathrm{BMC}$, research is always in progress.
Learn more biomedcentral.com/submissions 